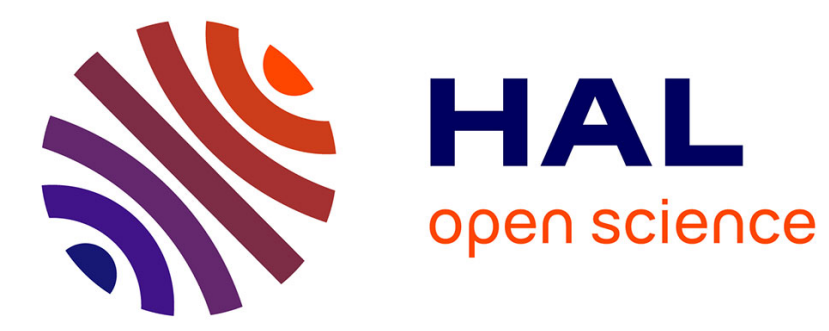

\title{
The two dimensional electron solid charge density wave
} F. Williams

\section{To cite this version:}

F. Williams. The two dimensional electron solid charge density wave. Journal de Physique IV Proceedings, 1993, 03 (C2), pp.C2-3-C2-8. 10.1051/jp4:1993201 . jpa-00251570

\section{HAL Id: jpa-00251570 https://hal.science/jpa-00251570}

Submitted on 1 Jan 1993

HAL is a multi-disciplinary open access archive for the deposit and dissemination of scientific research documents, whether they are published or not. The documents may come from teaching and research institutions in France or abroad, or from public or private research centers.
L'archive ouverte pluridisciplinaire HAL, est destinée au dépôt et à la diffusion de documents scientifiques de niveau recherche, publiés ou non, émanant des établissements d'enseignement et de recherche français ou étrangers, des laboratoires publics ou privés. 


\title{
The two dimensional electron solid charge density wave
}

\author{
F.I.B. WILLIAMS
}

Laboratoire de Matière Condensée Quantique, Service de Physique de l'Etat Condensé, Commissariat d l'Energie Atomique, 91192 Gif-sur Yvette, France

\begin{abstract}
A brief presentation of the physics and physical realisations of classical and quantum 2 dimensional electron solids is followed by a tourist guide to some of the experiments.
\end{abstract}

\section{Introduction}

A 2D electron solid (ES) can be thought of as a charge density wave (CDW) with $\sim 100 \%$ modulation amplitude. Like a CDW, it has internal dynamics, usually described in terms of phonons rather than phasons. Like a CDW, it is unstable to a random field and becomes pinned to the host disorder with attendant threshold for conduction. Unlike the customary CDW, the ES is stabilised by Coulomb interaction alone and the host only intervenes through the dielectric constant $\varepsilon$ and the effective mass $\mathrm{m}^{*}$.

\section{Physical realisations}

Fig.1 illustrates the two systems which have been most studied: electrons on liquid helium, well described by classical statistics, and electrons at an epitaxial semiconductor heterojunction requiring quantum statistics. In both cases electrons accumulate at the interface under the combined action of a perpendicular electric field and a difference in dissolution energy between the two milieux. An essential advantage of the 2D configuration is that the charge compensation can be set back into the third dimension to reduce its contribution to the disorder field (capacitor plate or remote ionised

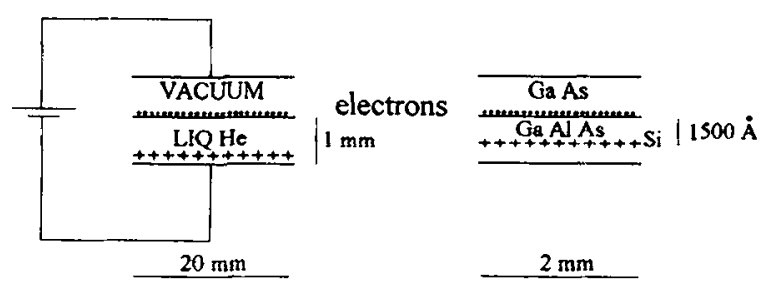

$$
\begin{gathered}
10^{7}<n_{s}<10^{9} \mathrm{~cm}^{-2} \\
\varepsilon \approx 1 \mathrm{~m}^{*} \approx m \\
10^{-1}<T_{m}<1 \mathrm{~K}
\end{gathered}
$$

$$
\begin{gathered}
4.10^{10}<n_{s}<4.10^{11} \mathrm{~cm}^{-2} \\
\varepsilon \approx 13 \mathrm{~m}^{*} \approx 0.07 \mathrm{~m} \\
10^{-2}<T_{m}<1 \mathrm{~K} \\
8<B_{C}<80 \text { Tesla }
\end{gathered}
$$

Fig.1. Structure of the two most exploited means of producing an electron system with two dimensional dynamics and low disorder.

\section{Ingredients}

Thermal and zero-point fluctuation energies $W_{t}=k_{B} T$ and $W_{q}=\hbar^{2} / m^{*} a^{2}\left(\pi \cdot a^{2}=n_{s}\right.$ the areal number density of electrons), favour the delocalisation of a liquid-like configuration whereas the correlation energy $\sim V_{c}=e^{2} / \varepsilon . a$ resulting from the Coulomb interaction is minimised by a periodic localised charge distribution. In the quantum limit, the effect of a magnetic field on the electron 
dynamics is to supress the thermal and zero-point delocalising actions. At zero temperature and magnetic field, the ratio $V_{c} / W_{q}=e^{2} m^{*} \cdot a / \varepsilon \cdot \hbar^{2}=a / a_{B}=r_{s}$ determines the state of the system. As $r_{s} \geq r_{s}(W 2 d) \approx 37$ [1], the ground state becomes crystalline rather than fluid: this is the true Wigner transition [2].

What have been seen are the thermally induced low density electron crystal to liquid transition and the magnetically induced quantum liquid to solid transition at high perpendicular magnetic field. In the clasical limit, the density is too low for $W_{q}$ to play a rôle and the play-off is between $T$ and $V_{c}$, expressed by the ratio $\Gamma=V_{c} / k_{B} T$ which determines the state of the system: crystalline for $\Gamma>\Gamma_{m} \approx 127\left(T_{m} \approx 0.9 \mathrm{~K}\right.$ for $n_{s} \approx 10^{9} \mathrm{~cm}^{-2}$ for electrons on helium).

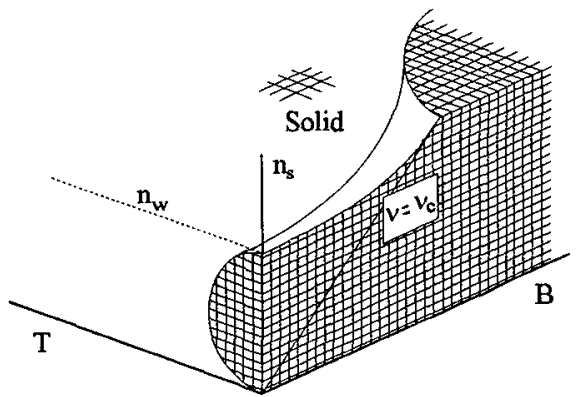

Fig.2. Schernatic phase diagram for interacting electrons with two dimensional dynamics and zero random field to show liquid-solid phase boundary as a function of areal number density $n_{s}$, temperature $T$ and perpendicular magnetic field $B$. Indicative number are:

$n_{W} \approx 10^{13} \mathrm{~cm}^{-2}$ for $\mathrm{m}^{*}=m, \varepsilon=1$ and $a_{B} \approx 0.5 \AA$ (electrons on helium) $n_{W} \approx 2.10^{8} \mathrm{~cm}^{-2}$ for $m^{*} \approx 0.07 m, \varepsilon \approx 13$ and $a_{B} \approx 100 \AA$ (electrons in GaAs); $v_{c} \approx 0.19$ corresponding to 20 Tesla for $n_{s}=10^{11} \mathrm{~cm}^{-2}$.

The section $n_{s}=$ const $\gg n_{W}=1 / \pi \cdot r_{s}^{2}(W 2 d)$ is appropriate to a typical experiment for the GaAs system: when expressed in reduced variables $(v, t)$ it should be independent of $n_{s}$ (see text and Fig.5)
In the extreme quantum limit, where $r_{s} \ll<r_{s}(W 2 d)$, the system is a quantum liquid (metal) in zero field. In increasing perpendicular magnetic field $B$ at low temperature, the system displays first the quantum Hall effects situated by the value of the "Landau level filling factor" $v=n_{s} \Phi_{0} / B$, the number of electrons per flux quantum $\Phi_{0}=h c / e$. In a non-interacting picture, when a Landau level is incompletely filled there remains a choice in the way the elecrons are distributed without altering the kinetic energy. In the presence of interaction, the system can take advantage of this choice to build in correlation between the particles to minimise the Coulomb energy. The fractional quantum Hall effect results from this electron correlation in the quantum liquid state. As the filling factor becomes smaller yet, correlations become more important until finally they take on long range character as electron orbits become centred on lattice points and the system acquires the internal mechanical rigidity of a solid. By extension from the Wigner transition, this phase is referred to as the Magnetically Induced Wigner Solid (MIWS).

A simple geometrical picture consists of distributing the electrons of average separation $a$ among the available states of radius $\ell_{c}=\sqrt{\hbar c / e B}$ (the quantum cyclotron radius), each of which occupies the area of a flux quantum and which just fill the plane. The freedom of choice grows as $\ell_{0}$ is made smaller until $a^{2} / \ell_{c}^{2}=2 / v \geq 2 / v_{c}$ when a crystal latttice distribution is the most favorable $\left(v_{c} \approx 0.19\right.$, corresponding to $B \approx 20$ Tesla for $n_{s} \approx 10^{11} \mathrm{~cm}^{-2}$ ). The correlation energy $\sim V_{c}$ stabilising this configuration must be compared to the destabilising thermal agitation $T$. The phase diagram is expected to take a universal form in the reduced variables $\left(v, T / V_{c}\right)$. Since in infinite field the electron cyclotron radius shrinks to a point and one expects the melting temperature to take its classical value $T_{c m}=V_{c} / \Gamma_{m}$, it is convenient to normalise the reduced temperature variable to $t=T / T_{c m}\left(T_{c m} \approx 0.6 \mathrm{~K}\right.$ for $n_{s} \approx 10^{11} \mathrm{~cm}^{-2}$ in $\mathrm{GaAs}$ ). This offers a simple and important test for any interpretation in terms of a MIWS: at densities sufficiently higher than the zero-field Wigner transition density, the phase diagram 
for any fixed $n_{s}$ should be the same in the reduced variable representation $(v, t)$ with asymptote $t \rightarrow 1$ as $v \rightarrow 0$. A schematic form of the phase diagram is shown in Fig. 2 .

Mechanical properties: phonons, transport and pinning

In the unidimensional CDW, phase rigidity is described by a sole longitudinal elastic constant. In a two dimensional CDW or ES, on the other hand, one requires a longitudinal and a transverse elastic constant to write the free energy in terms of the lattice strain. The dynamics is described by two phonon excitation branches. The longitudinal branch is given by $\omega_{\ell}^{2}=2 \pi e^{2} n_{s} q / m^{*} \varepsilon$ for unscreened plasmons of wavevector $q[3]$, reflecting the size dependent nature of Coulomb compressibility (capacitance has units of length): it is essentially the same for a liquid as for a crystal. The transverse branch, on the other hand, is radically different in the two configurations. A shear deformation encounters only a velocity dependent force in the liquid, resulting in a damped excitation branch around zero frequency, whereas in the solid it is elastically opposed by the variation in correlation energy to give rise to a finite frequency transverse phonon branch

$$
\omega_{t}^{2}=\beta e^{2} q^{2} / \varepsilon . m^{*} a \approx q a . \omega_{l}(q),
$$

where $\beta \approx 1$, reflecting the short range, intrinsic, nature of the shear stress. In a magnetic field, these two branches are coupled by the Lorentz force which, although it depresses the lower excitation branch, leaves it with a finite frequency and no gap $\left(\omega_{+}^{2} \approx \omega_{c}^{2}+\omega_{l}^{2}\right.$ and $\omega_{-}^{2} \approx \omega_{l} \omega_{t} / \omega_{c}$ if $\omega_{c}>\omega_{l}$ where $\omega_{c}=e B / \mathrm{m}^{*} c$, the cyclotron frequency). The existance of a finite frequency lower phonon branch is a sign of the elastic shear rigidity characteristic of a solid phase.

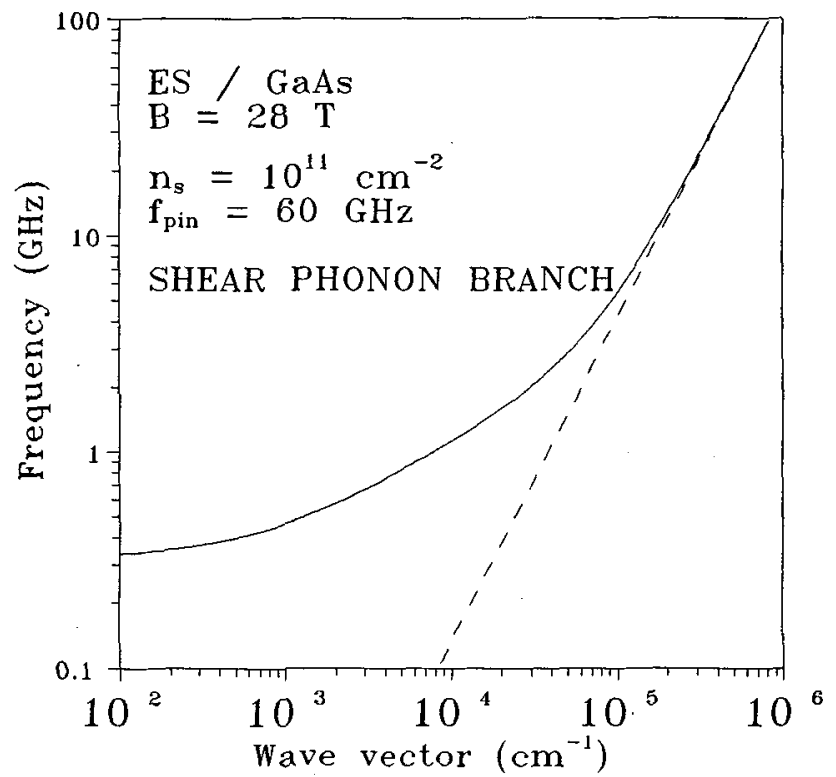

Fig.3a. Dispersion of lower hybrid mode of a pinned electron solid in condition typical of experiments on electrons at GaAs/GaAlAs heterojunction. Dashed asymptote correspond to $f_{p i n}=0$ This branch does not exist in the fluid phase.

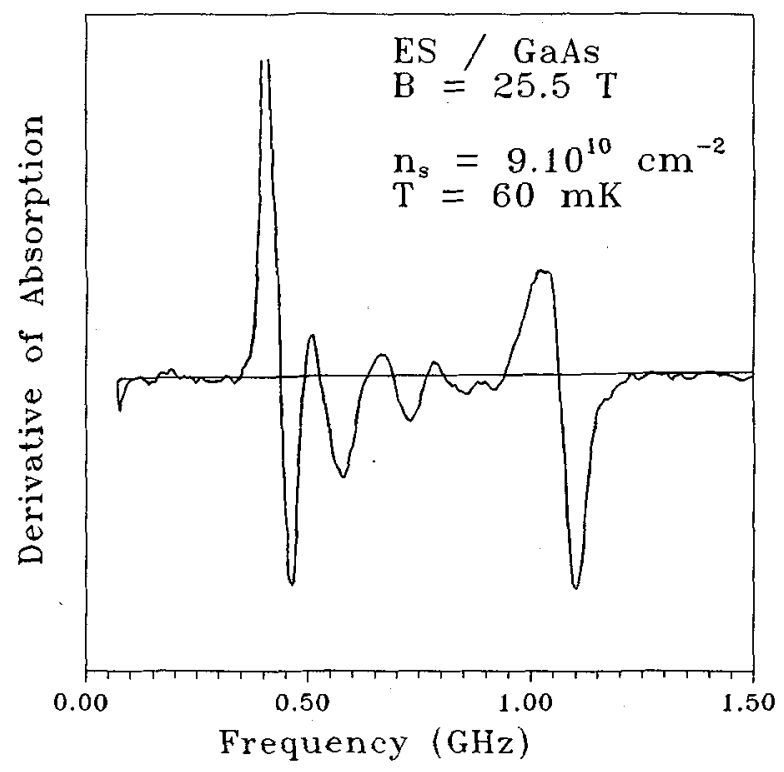

Fig.3b. Example of an absorption spectrum from a radiofrequency electric ficld of 16 micron periodicity in the high field, low temperature (solid) portion of the phase diagram. 
Transport is governed by the resistance opposing a global displacement of the system with respect to the milieu in which it is embedded. In the absence of disorder, only inertia opposes this displacement, whatever the internal structure. The situation is different in the presence of host disorder. An independent electron scatters off the roughness of the random field of the disorder: if it is not strongly localised, its velocity in an in-plane field is described by a mobility. In an interacting system, an internal deformation accompanies the motion: in a liquid, where shear stress is velocity dependent, a global displacement of the fluid is again described by a mobility which now includes the internal shear forces required to flow around "obstacles" of the random field. The situation is again radically different for a solid: the instability of long range translational order to a random field [4] manifests itself, as in CDW systems, by the crystal breaking into domains whose size is determined by the compromise between elastic deformation energy (compression in 1D, shear in 2D because of the relative smallness of the shear modulus for Coulomb interactions). Because the relaxed solid requires a minimum force to slide with respect to the random field, the liquid to solid transition results in a conductor to insulator transition. The minimum field $E_{T}$ for sliding conduction gives an estimate of the restoring force constant $\kappa \approx e . E_{T} / a$ per electron. This suggests a relationship between $E_{T}$ and the pinning frequency whose experimental validity provides a check on the model: $\omega_{p i n}=\sqrt{\frac{\kappa}{m^{*}}} \approx \sqrt{\frac{e E_{T}}{a m^{*}}}$.

The pinning modifies the phonon dispersion. The extra restoring force of the pinning must be added to the internal restoring force: $\omega_{l, t}^{2} \rightarrow \omega_{l, t}^{2}+\omega_{\text {pin }}^{2}$ in the expressions for the phonon frequencies, resulting in a gap $\omega_{p \text { in }}^{2} / \omega_{c}$ as $q \rightarrow 0$ in the lower hybrid branch as shown in Fig.3. These aspects and the effect of screening by quasi-free electrons not locked into the lattice, are dealt with more thoroughly in the following article by Littlewood.

\section{Experimental evidence}

The classical, low density, electron solid was observed first for electrons on liquid helium [5]. The electron correlation in the crystalline phase allows the helium surface to take on the imprint of the electron distribution and thereby participate in the electron motion [6]. In this situation, the electron crystal can be used as a spatially periodic transducer for ripplons and resonance frequencies can be

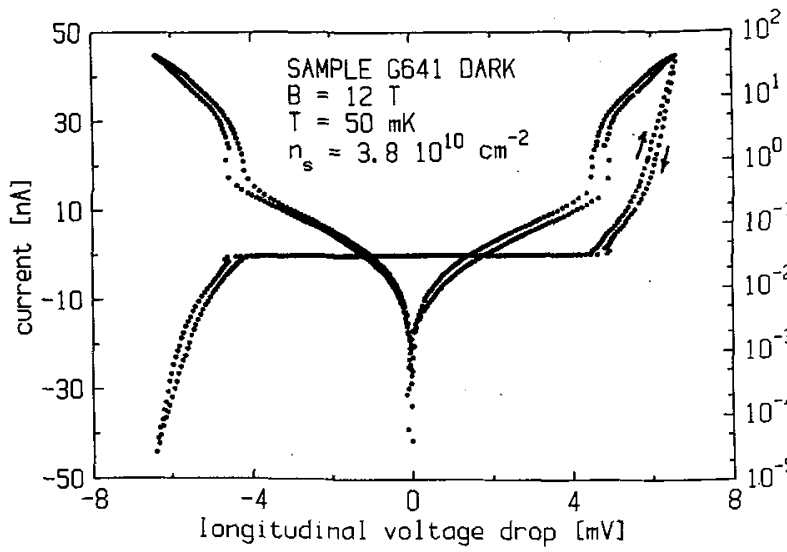

Fig.4a. A typical current-longitudinal voltage characteristic for the pinned solid in GaAs/GaALAs heterojunction: $D C$ scan over $\sim 100 \mathrm{sec}$ and electrometer (EL) voltage measurement. The centrally flat curve is a linear (left scale) and the cusped curve is a log (right scale) representation of the current. The reduced variable coordinates on the universal phase diagram of Fig. 5 are $(v=0.13, t=0.14)$ related through the ripplon dispersion relation to the wavenumbers of the electron reciprocal lattice. The electron crystal is thus revealed to be hexagonal. Other experiments demonstrate the appearance of propagating transverse phonons whose dispersion relation has a gap determined by the coupling to the surface and an asymptotic slope (propagation velocity) from which the shear modulus is determined [7]. The relationship of the latter to the melting temperature suggested strongly that melting proceeds via dislocation pair unbinding, an idea further reinforced by the experiments on the specific and latent heats [8]. All these experiments could be analysed in terms of a uniform twodimensional milieu. It is however possible to impose a roughness by thinning the liquid helium pad separating the electrons from the 
bottom of the containing structure: the effects of such a random field on the conductivity are the subject of the article by Dahm in this same issue [9].

The electron crystal on liquid helium is thus seen to be a particularly versatile experimental realisation of a two-dimensional CDW where the interaction with the deformable but nonetheless uniform substrate mimics a commensurate pinning potential and phason dynamics on which it is possible to impose a random field whose strength can be varied in situ.

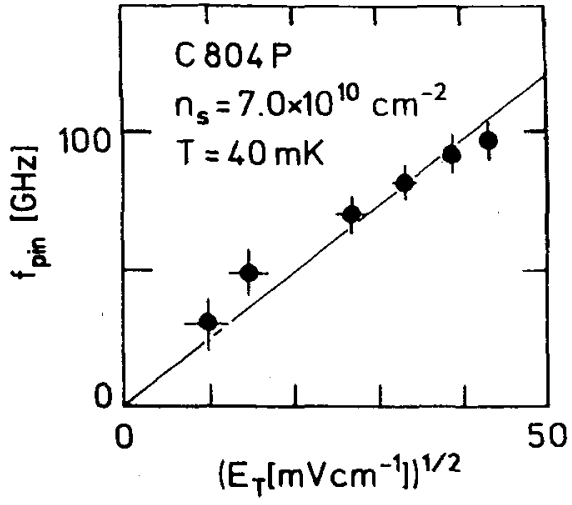

Fig4b. Pinning frequency deduced from if spectrum plotted against simultaneously measured conduction threshold $E_{T}$. The relationship suggested in the text seems quite well obeyed.
Evidence of a two-dimensional electron solid in the quantum limit first appeared for electrons at a high quality GaAs/GaAlAs epitaxial heterojunction: low lying finite frequency magneto-phonon excitations are observed to appear at low temperature and high perpendicular magnetic field [10] (Fig.3b). The loci of appearance for different density samples $\left(2<r_{s}<4\right)$ are coherent with a universal phase diagram in $(v, t)$ reduced variables. The frequencies observed are of the right order of magnitude for shear excitations on the micron length scales of the experiment, but they indicate a small gap in the dispersion relation and do not show the scaling with density expected of an ideal uniform system. This phenomenon is accompanied by a qualitative change in the temperature

dependence of the longitudinal resistance $(V / I)$ at constant current [11]. Further experiment showed concommitant current voltage non-linearity $[12,13]$ (Fig.4a). The voltage threshold for conduction is found to be quantitatively related to the magneto-phonon frequencies, giving strong support to the interpretation of the gap in terms of pinning [13] (Fig.4b). Experiments using surface acoustic waves have also shown new excitations to appear in the solid phase with similar pinning frequencies as found by direct electromagnetic absorption [14].

The availability of better samples revealed a more complex phase diagram than originally thought, with reentrant portion(s) indicative of competition with the strongly correlated liquid at certain rational filling factors. The photoluminescence (PL) spectrum accompanying electron recombination with a free hole [16] and with an acceptorbound hole [15] also indicates the appearance of a new phase. Time resolved PL experiments indicate a slower recombination rate in the solid phase, compatible with the idea that the recombination rate with electrons locked into a solid network pinned to the milieu is slower than for electrons in a mobile delocalised liquid phase [17]. Fig. 5 gives a schematic representation of the phase diagram in $(v, t)$ variables: all the different techniques give the same diagram to within experimental uncertainties, with the exception of the reentrant portion at

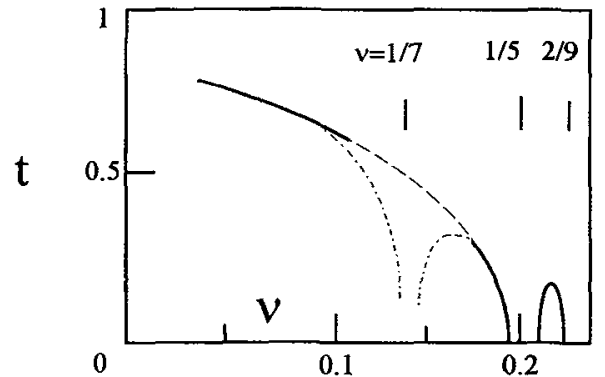

Fig.5. A schematic phase diagram in reduced variable $(v, t)$, as explained in the text, for a large variety of high density $\left(2<r_{s}<4\right)$ samples of electrons at GaAs/GaAlAs heterojunctions. This is a composite of magnetophonons $[10,14]$, non-linear conduction $[12,13]$ and photoluminescence [15,16,17] results. Only the latter [17] show a clear cusp at $v=1 / 7$. There are small discrepancies in the temperature scale between different groups, but these are probably within experimental uncertainties. 
$v=1 / 7$ found in the time resolved photoluminescence but not seen in transport or magneto-phonons.

The picture emerging here is that of a crystal broken into decorrelated domains of several hundreds of electrons by the action of the random field produced by imperfections of the milieu. It is an example of a two-dimensional incommensurably pinned CDW. It can be hoped that the present intense activity will lead to a better understanding of the conduction mechanism, the melting mechanism and the approach to the zero-field Wigner transition (by investigating systems of higher like electrons in $\mathrm{Si}$ MOSFET structures [18] or holes at the GaAs/GaAlAs heterojunctions [19].

\section{Acknowledgements}

As nominal author of this contribution, I am to blame for any misrepresentation of what is essentially a collective effort. It is my pleasure to acknowledge the direct collaboration of E.Andrei, R.Clark, G.Deville, B.Etienne, T.Foxon, C.Glattli, J.Harris, D.Marty, E.Paris, F.Perruchot, J.Poitrenaud and P.Wright; stimulating discussions with A.Janossy and G.Mihaly on CDW aspects; and stimulus from numerous "competitors".

\section{References}

[1] Tanatar B. and Ceperley D.M., Phys. Rev. B39 (1989) 812.

[2] Wigner E.P., Phys. Rev.46 (1934) 1002.

[3] Chaplik A.V., Zh. Eksp. Teor. Fiz. 62 (1972) 746 (Sov. Phys. JETP 35 (1972) 392).

[4] Imry Y. and Ma S., Phys. Rev. Lett., 35 (1975) 399.

[5] Grimes C.C. and Adams G., Phys. Rev. Lett. 42 (1979) 795.

[6] Fisher D., Halperin B.I. and Platzman P.M., Phys. Rev. Lett. 42 (1979) 798.

[7] Deville G., Valdes A., Andrei E. and Williams F.I.B., Phys. Rev. Lett. 53 (1984) 588.

[8] Glattli C., Andrei E. and Williams F.I.B., Phys. Rev. Lett. 60 (1988) 420.

[9] Jiang H.W. and Dahm A.J., Phys. Rev. Lett. 62 (1989) 1396.

[10] Andrei E.Y., Deville G., Glattli D.C., Williams F.I.B., Paris E., and Etienne B., Phys. Rev. Lett. 60 (1988) 2765; Glattli D.C., Deville G., Dubureq V., Williams F.I.B., Paris E., Etienne B. and Andrei E.Y., Surf. Sci. 229 (1990) 344.

[11] Willett, R.L., Stormer H.L., Tsui D.C., Pfeiffer L.N., West K.W. and Baldwin K.W., Phys. Rev. B38 (1988) 7881; Jiang H.W., Willett R.L., Stormer H.L., Tsui D.C., Pfeiffer L.N. and West K.W., Phys. Rev; Lett. 65 (1991) 633.

[12] Goldman V.J., Cunningham J.E., Shayegan M. and Santos M., Phys. Rev. Lett. 65 (1990) 2188; Williams F.I.B., Andrei E.Y., Clark R.G., Deville G., Etienne B., Foxon C.T., Glattli D.C., Paris E. and Wright P.A. in "Localization and Confinement of Electrons in Semiconductors" Springer-Verlag (1990) 192; Y. P. Li, T. Sajoto, L. W. Engel, D. C. Tsui, and M. Shayegan, Phys. Rev. Lett. 67, 1630 (1991).

[13] Williams F.I.B., Wright P.A., Clark R.G., Andrei E.Y., Deville G., Glattli D.C., Probst O., Etienne B., Dorin C., Foxon C.T. and Harris J.J., Phys. Rev. Lett., 66 (1991) 3285.

[14] Paalanen M.A., Willett R.L., Ruel R.R., Littlewood P.B., West K.W. and Pfeiffer L.N., Phys. Rev. B45 (1992) 13784.

[15] Buhmann H., Joss W., von Klitzing K., Kukushkin I.V., Martinez G., Plaut A.S., Ploog K. and Timofeev V.B., Phys. Rev. Lett. 66 (1991) 962.

[16] Goldys E.M., Brown S.A., Dunford R., Davies A.G., Newbury R., Clark R.G., Simmonds P.E., Harris J.J. and Foxon C.T., Phys. Rev. B46 (1992) 7957.

[17] Kukushkin I.V., Pulsford N.J., von Klitzing K., Haug R.J., Ploog K. and Timfeev V.B., EuroPhys. Lett. 23 (1993) 211.

[18] Pudalov V.M., D'Iorio M., Kravchenko S.V. and Campbell J.W., Phys. Rev. Lett. 70 (1993) 1866.

[19] Santos M.B., Suan Y.W., Shayegan M., Li Y.P., Engel L.W. and Tsui D.C., Phys. Rev. Lett. 68 (1992) 1188. 